\title{
Bionic Motion Detection Applied in Surveillance of Airport
}

\author{
Huabo Sun ${ }^{1}$, Yan $\mathrm{Ma}^{1}$, Gaojian Kang${ }^{2}$, Xiaoming $\mathrm{Ma}^{1}$
}

1. China Academy of Civil Aviation Science and Technology, Beijing 100028, P.R.China

2. Beijing Institute of Astronautical Systems Engineering, Beijing 100076, P.R.China

sunhb@mail.castc.org.cn

Keywords: bionic motion detection, security monitoring, detection algorithm, image matching.

\begin{abstract}
Conventional security monitoring of civil airport usually uses fixed cameras to acquire images. There are several problems with performance including difficulties introduced in the information transmission, storage, and analysis stages of the process. Insect compound eyes offer unique advantages for moving target capture and these have attracted the attention of many researchers in recent years. This paper contributes to this research by proposing a new bionic processing mode and algorithm applying it in security monitoring of civil airport. Image matching is also illustrated with different conditions. This mode and algorithm effectively achieve safety surveillance of airport with large field of view and high real-time processing.
\end{abstract}

\section{Introduction}

Moving target detection has been applied in many applications, such as video surveillance of civil airport, in recent years. Traditional fixed camera systems for surveillance dealt with the acquired images have two main questions, one is that the field of view is limited, and the other is that data storage is too large with the accumulation. During process acquired image, we must transform the three dimensional objects, which are acquired by high resolution sensors, to two dimensional images. Following this task one must then distinguish and locate the targets using signal-processing techniques. Finally we reverse the two-dimensional information to three-dimensions in order to redisplay the actual objects. The precision of detection and the amount of calculation required are positively correlated in such transformations. The higher the precision required the greater the amount of data involved. Consequently this delivers a less than real-time performance. As a result of these tradeoffs many researchers have turned their attention to a new image acquisition and processing mode called bionic compound eyes ${ }^{[1]}$. Tanida ${ }^{[2]}$ et al proposed a bionic compound eyes imaging system called TOMBO (Thin Observation Module by Bound Optics) which adapted micro lens and photo detectors for the acquisition and receiving assembly of light signals. This information is then processed to restructure the targets. Duparre ${ }^{[3]}$ et al proposed and manufactured the AACO (Artificial Apposition Compound Eye Objective) ${ }^{[4]}$. Insect compound eyes have many advantages in moving target detection. They can detect and recognize targets in large areas using minimal information obtained through feature outline.

The remainder of this paper is organized as follows. In section 2 we analyze the bionic mechanism. Section 3 provides bionic detection model. In section 4 bionic detection algorithm is discussed based on different image matching. Section 5 closes the paper with some conclusions and directions for future research.

\section{Detection mechanism}

Stephen A. Baccus et al discovered that there were two types cell existed in compound eyes, one is polyaxonal amacrine cell, and the other is ganglion cell. Different cell plays different roles in target detection. Based on their long-term research, they proposed compound eyes detection model ${ }^{[5]}$, show as Fig.1. 


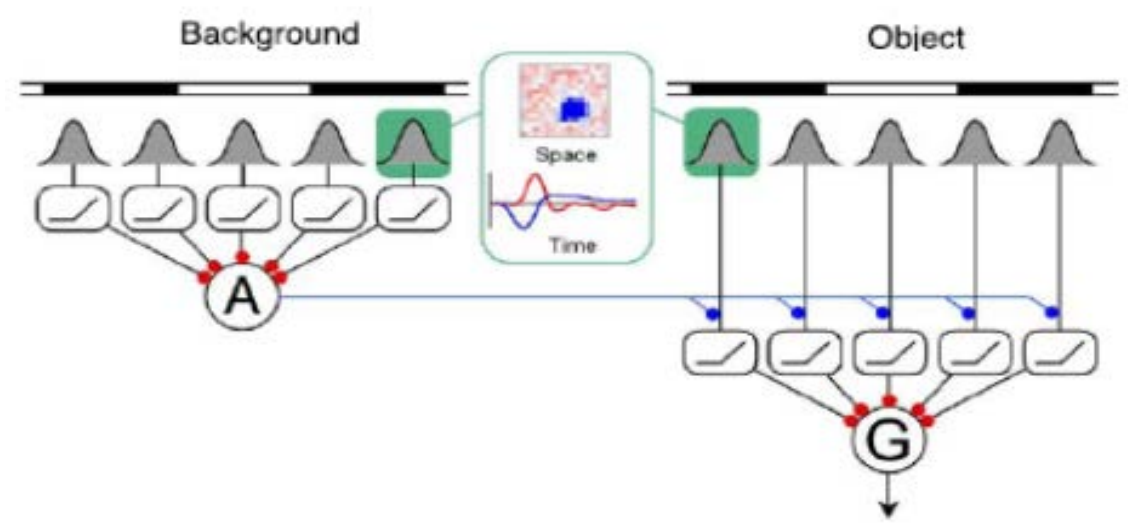

Fig. 1 The Baccus improved model.

Reichardt et al proposed that there are two independent channels in compound eyes ${ }^{[6]}$. One is called the "large field system" and the other is called the "small field system". The large field system detects global motion and the small field detects moving targets accurately. Insects can maintain high-resolution views of their targets while at the same time maintaining information about other targets in the same view. The mechanism of the compound eyes provides a solution to the tradeoffs between viewing field size, resolution, and real-time processing. These are very important problems that must be addressed when developing moving target detection systems.

\section{Bionic detection model}

On the basis of compound eye model we have proposed a bi-level contour detection model with variable resolution bionic compound eyes, as is shown in Fig. 2.

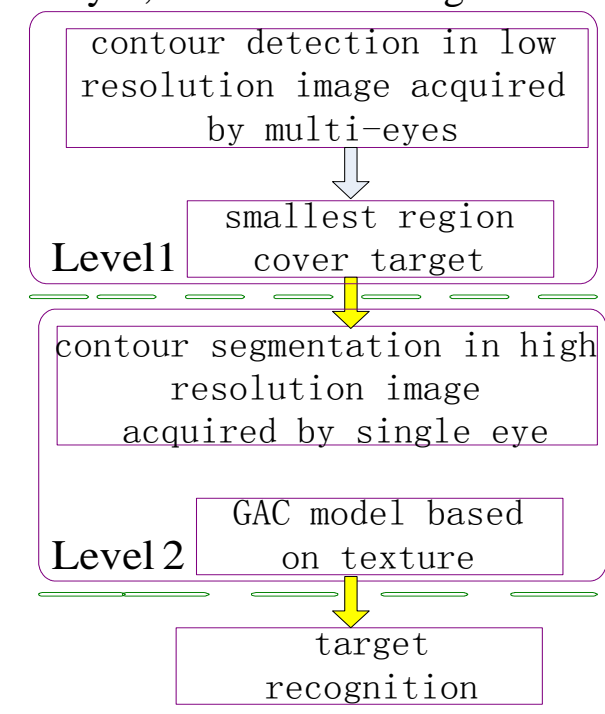

Fig. 2 Flowchart diagram of the bi-level contour detection model.

There are three important components of this model. Firstly there is contour detection which is applied to the lowest resolution images. When this is complete the texture gradient computation is performed by conducting contour segmentation on the high resolution image(s) acquired by the single eye. Finally a Geodesic Active Contour (GAC) model for texture boundary detection is applied from which the moving target is recognized.

Our bionic compound eyes model includes two components: bionic structure, and bionic function. The biometric structure is the foundation component which can acquires data with large view of field. The bionic function performs the detection of moving targets. The region of motion is computed by the bionic function. The final component of our bionic eyes mechanism is executed after contour segmentation of the remotely sensed images. The moving target is then extracted. It is clear that biometric level grows from low-level complexity to the highest level of complexity when the moving target is extracted. 


\section{Bionic detection algorithm}

Based on the mechanism of insect compound eyes for detecting moving targets, we have constructed a bionic compound eyes detection system ${ }^{[7]}$. Different from conventional data acquisition, the bionic mechanism provides a new solution to acquire data that combines high and low resolution cameras. Our compound eyes device consists of seven cameras. Using a servo system to control the angle of the cameras we can control the cameras to not only point to a specific position but also to monitor large areas in several directions. During data acquire and matching, there are two conditions, as is shown in the following.

4.1 Different image matching

\subsubsection{Consistent camera angles}

Image acquisition and matching, with consistent camera angles, is show as Fig. 3.

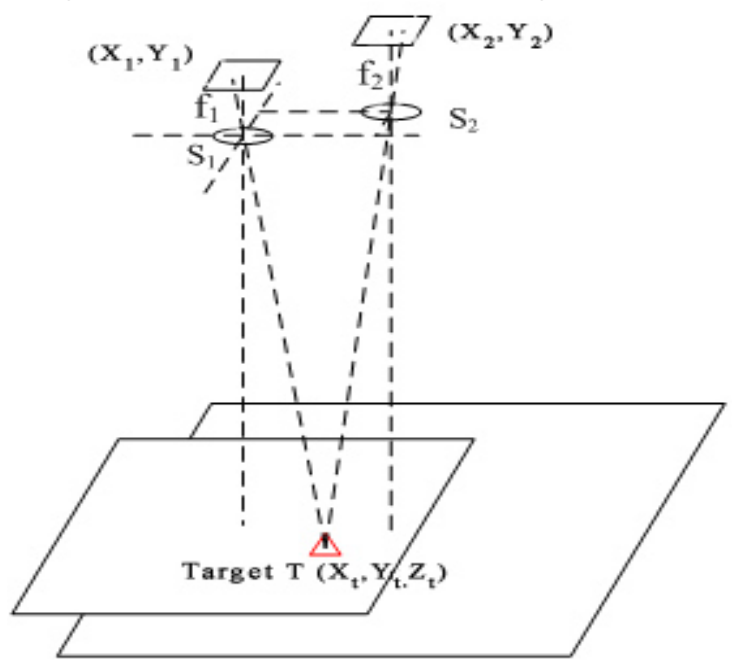

Fig. 3 Photo consistent camera angles.

In the Fig. $3, S_{1}$ indicates the center camera with high-resolution, $S_{2}$ indicates the any adjacent camera with low-resolution, $\left(x_{2}, y_{2}\right)$ stands for image coordinate in low-resolution camera, $\left(x_{1}, y_{1}\right)$ stands for image coordinate in high-resolution camera, $\left(X_{t}, Y_{t}, Z_{t}\right)$ presents coordinate of target $\mathrm{T}$, f stands for focal length. During data acquisition, low-resolution camera detects target profile, and gets target position, and then high-resolution camera adjusts to image with the same position. When we take photo, target point, center of photography and the image point are in a straight line. According to the collinear three points, we can get the formula, described as

$$
\begin{aligned}
& \frac{X_{t}-X_{1}}{x_{1}}=\frac{Y_{t}-Y_{1}}{y_{1}}=\frac{Z_{t}-Z_{1}}{-f_{1}}=\frac{1}{m_{1}} \\
& \frac{X_{t}-X_{2}}{x_{2}}=\frac{Y_{t}-Y_{2}}{y_{2}}=\frac{Z_{t}-Z_{2}}{-f_{2}}=\frac{1}{m_{2}}
\end{aligned}
$$

Coarse matching with different resolution images means to deduce $\left(x_{1}, y_{1}\right)$ from $\left(x_{2}, y_{2}\right)$. When photography scale $\mathrm{m}$ is known, we can calculate $\left(x_{1}, y_{1}\right)$ with equation

$$
\begin{aligned}
& x_{1}=m_{1}\left(\frac{x_{2}}{m_{2}}+X_{2}-X_{1}\right) \\
& y_{1}=m_{1}\left(\frac{y_{2}}{m_{2}}+Y_{2}-Y_{1}\right)
\end{aligned}
$$

\subsubsection{Tilted camera angles}

Image acquisition and matching, with tilted camera angles, is show as Fig. 4. 


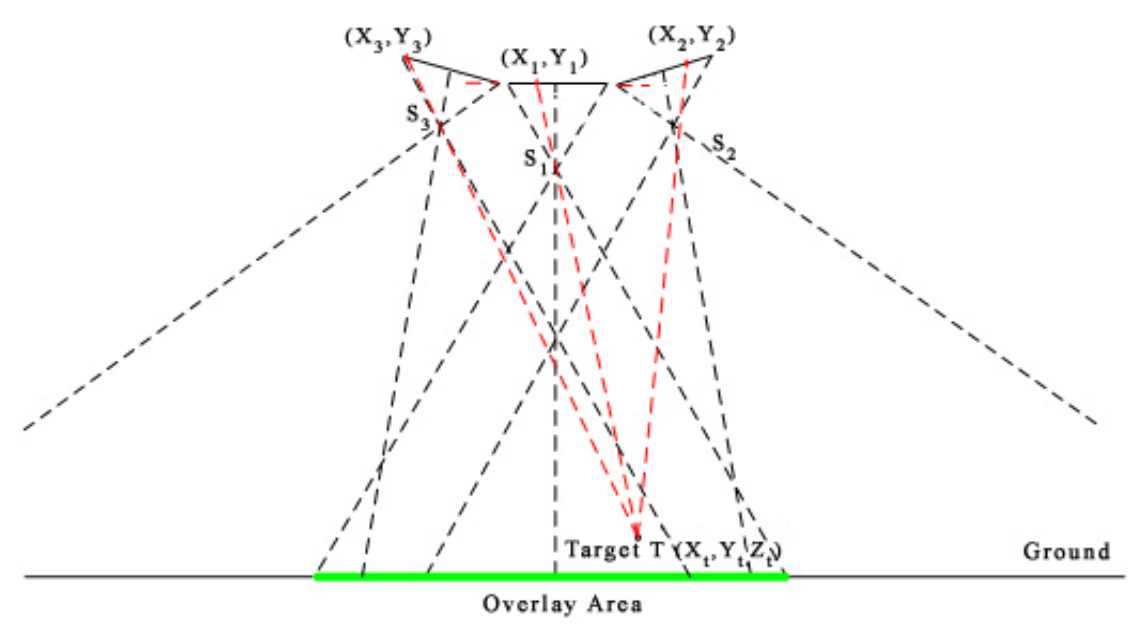

Fig. 4 Photo tilted camera angles.

In the Fig. $4, S_{1}$ indicates the center camera with high-resolution, $S_{2} \& S_{3}$ indicate the any adjacent camera with low-resolution, $\left(X_{t}, Y_{t}, Z_{t}\right)$ presents coordinate of target $\mathrm{T}$. According to the collinear three points, we can also get the formula, described as

$$
\frac{X}{X_{t}-X_{S}}=\frac{Y}{Y_{t}-Y_{S}}=\frac{Z}{Z_{t}-Z_{S}}=\frac{1}{\lambda} \text {. }
$$

For camera $S_{2} \& S_{3}$, we can separately give collinearity equation (4) and (5).

$$
\begin{aligned}
& x_{2}=-f_{2} \frac{a_{1}^{2}\left(X_{t}-X_{S 2}\right)+b_{1}^{2}\left(Y_{t}-Y_{S 2}\right)+c_{1}^{2}\left(Z_{t}-Z_{S 2}\right)}{a_{3}^{2}\left(X_{t}-X_{S 2}\right)+b_{3}^{2}\left(Y_{t}-Y_{S 2}\right)+c_{3}^{2}\left(Z_{t}-Z_{S 2}\right)} \\
& y_{2}=-f_{2} \frac{a_{2}^{2}\left(X_{t}-X_{S 2}\right)+b_{2}^{2}\left(Y_{t}-Y_{S 2}\right)+c_{2}^{2}\left(Z_{t}-Z_{S 2}\right)}{a_{3}^{2}\left(X_{t}-X_{S 2}\right)+b_{3}^{2}\left(Y_{t}-Y_{S 2}\right)+c_{3}^{2}\left(Z_{t}-Z_{S 2}\right)} \\
& x_{3}=-f_{3} \frac{a_{1}^{3}\left(X_{t}-X_{S 3}\right)+b_{1}^{3}\left(Y_{t}-Y_{S 3}\right)+c_{1}^{3}\left(Z_{t}-Z_{S 3}\right)}{a_{3}^{2}\left(X_{t}-X_{S 3}\right)+b_{3}^{2}\left(Y_{t}-Y_{S 3}\right)+c_{3}^{2}\left(Z_{t}-Z_{S 3}\right)} \\
& y_{3}=-f_{3} \frac{a_{2}^{3}\left(X_{t}-X_{S 3}\right)+b_{2}^{3}\left(Y_{t}-Y_{S 3}\right)+c_{2}^{3}\left(Z_{t}-Z_{S 3}\right)}{a_{3}^{3}\left(X_{t}-X_{S 3}\right)+b_{3}^{3}\left(Y_{t}-Y_{S 3}\right)+c_{3}^{3}\left(Z_{t}-Z_{S 3}\right)}
\end{aligned}
$$

With photo camera, we can get rotation matrix of camera $S_{2} \& S_{3}$, described as $R_{2}, R_{3}$.

$$
R_{2}=\left[\begin{array}{lll}
a_{1}^{2} & a_{2}^{2} & a_{3}^{2} \\
b_{1}^{2} & b_{2}^{2} & b_{3}^{2} \\
c_{1}^{2} & c_{2}^{2} & c_{3}^{2}
\end{array}\right] \quad R_{3}=\left[\begin{array}{lll}
a_{1}^{3} & a_{2}^{3} & a_{3}^{3} \\
b_{1}^{3} & b_{2}^{3} & b_{3}^{3} \\
c_{1}^{3} & c_{2}^{3} & c_{3}^{3}
\end{array}\right] .
$$

With the equation (3), (4), (5), (6), we can calculate $\left(x_{1}, y_{1}\right)$, described as

$$
\begin{aligned}
& x_{1}=-f_{1} \frac{a_{1}^{1}\left(X_{t}-X_{S 1}\right)+b_{1}^{1}\left(Y_{t}-Y_{S 1}\right)+c_{1}^{1}\left(Z_{t}-Z_{S 1}\right)}{a_{3}^{1}\left(X_{t}-X_{S 1}\right)+b_{3}^{1}\left(Y_{t}-Y_{S 1}\right)+c_{3}^{1}\left(Z_{t}-Z_{S 1}\right)} \\
& y_{1}=-f_{1} \frac{a_{2}^{1}\left(X_{t}-X_{S 1}\right)+b_{2}^{1}\left(Y_{t}-Y_{S 1}\right)+c_{2}^{1}\left(Z_{t}-Z_{S 1}\right)}{a_{3}^{1}\left(X_{t}-X_{S 1}\right)+b_{3}^{1}\left(Y_{t}-Y_{S 1}\right)+c_{3}^{1}\left(Z_{t}-Z_{S 1}\right) .}
\end{aligned}
$$

\subsection{Contour detection object capture}

With our bionic compound eyes system, after we calibrate the cameras, and then we capture experimental data, shown as Fig. 5. According to the principle of binocular vision in computer vision and considering the actual, we select the photos taken by two low-resolution cameras as stereo images to construct three-dimensional. 

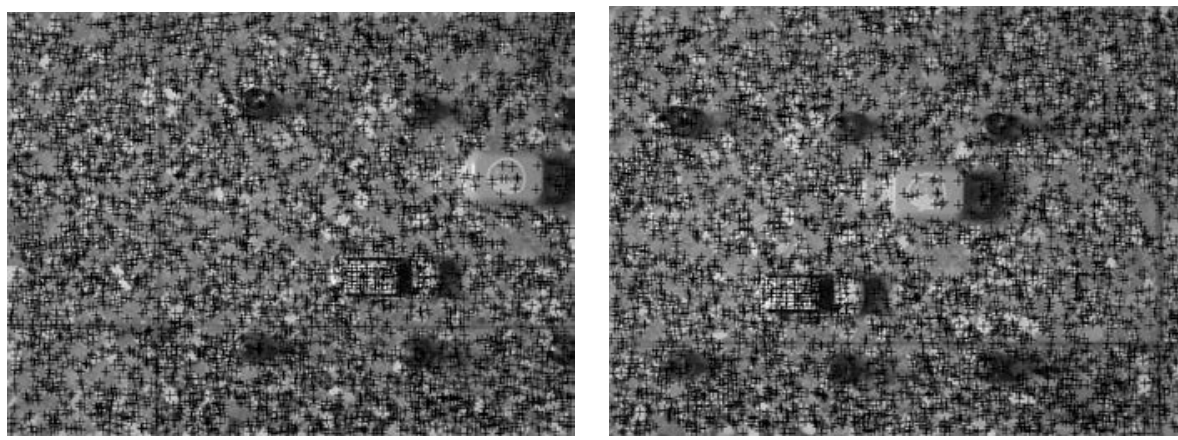

Fig. 5 The experimental data captured by compound eyes.

As the moving target and the surrounding environment can be expressed by feature point, then we extract feature points from stereo images and match them. We use SIFT algorithm to achieve feature extraction and RANSAC algorithm to achieve feature points matching. The result is shown as Fig. 6.

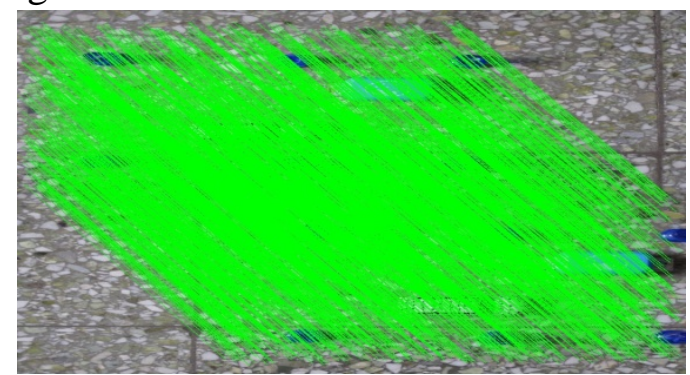

a. Extract and match of feature points.

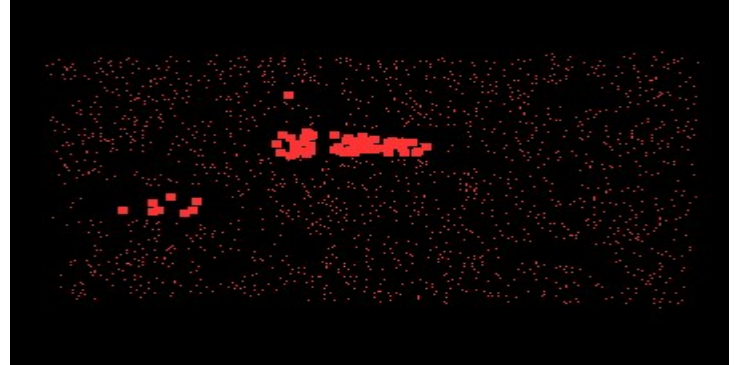

b. The point data of moving target and scene.

Fig. 6 Contour detection object capture.

After we have constructed 3D point cloud ${ }^{[8]}$, we should extract feature points of model car. According to experimental environment characteristics, we select the difference of height of the model car and other objects as criterion to distinguish the model car and other objects.

With bionic data acquisition mode, we can obtain several resolution images. We can detect target with high resolution and scene with low resolution; this will greatly increase the efficiency of the treatment.

\section{Conclusion}

This paper has carefully outlined the advantages of bionic motion detection for applying in Surveillance of civil airport. The paper has proposed detection mechanism and bionic algorithm of compound eyes. Different image matching is illustrated in detail. As discussed in the paper our method is simultaneously optimal in respect of the view field, resolution, and requirements for real-time processing. The approach of using both high and low resolution imagery in the moving object detection for security monitoring reduces the overall computation time. Subsequently, we believe that the overall computation time would be suitable for many civil airports and other squares. The work presented in this paper provides a novel resolution to security monitoring field of research.

\section{Acknowledgment}

The work presented in this paper is supported by National Science Foundation of China (ID: U1333124 \& U1433102).

\section{References}

[1] Ki-Hun Jeong, Jaeyoun Kim, Luke P. Lee, Biologically Inspired Artificial Compound Eyes, SCIENCE. 312(2006) 557-561.

[2] Yamada K, Ishida S, Shougenji R, Tanida J, Development of three dimensional endoscope by Thin Observation by Bound Optics(TOMBO), Automation Congress. (2006) 199-205. 
[3] Duparré J, Wippermann F, Dannberg P, Bräuer A, Artificial compound eye zoom camera, Bioinspiration biomimetics. 3(2008) 1806-1823.

[4] Duparre J, Radtke D, Bruckner A, et al, Latest developments in micro optical artificial compound eyes: a promising approach for next generation ultra-compact machine vision, SPIE. 6503(2007) 1-12.

[5] Stephen A. Baccus, Bence P.Ölveczky, Mihai Manu, Markus Meister. A retinal circuit that computes object motion [J]. Journal of Neuroscience, July 2, 2008, 28: 6807-6817.

[6] Reichardt W. Evaluation of optical motion information by movement detectors. Journal of Comparative Physiology A: Neuroethology, Sensory, Neural, and Behavioral Physiology, 1987, 161, 533-547.

[7] Huabo Sun, Haimeng Zhao and Peter Mooney et al, A Novel System for Moving Object Detection Using Bionic Compound Eyes, Journal of Bionic Engineering. 8(2011) 313-322.

[8] Huabo Sun, Boren Luo and Liling Yu et al, Mosaic research with 3D lidar point cloud of civil aircraft, 2013 2nd International Symposium on Instrumentation and Measurement, Sensor Network and Automation, IMSNA 2013, 683-686. 